\title{
Dynamical control of denaturation bubble nucleation in supercoiled DNA minicircles
}

\author{
François Sicard $\odot,{ }^{1, *}$ Nicolas Destainville, ${ }^{2}$ Philippe Rousseau, ${ }^{3}$ Catherine Tardin,${ }^{4}$ and Manoel Manghi $\odot^{2}$ \\ ${ }^{1}$ Department of Chemistry, King's College London, SE1 1DB London, United Kingdom \\ ${ }^{2}$ Laboratoire de Physique Théorique, IRSAMC, Université de Toulouse, CNRS, UPS, France \\ ${ }^{3}$ Laboratoire de Microbiologie et Génetique Moléculaires, Centre de Biologie Intégrative (CBI), Université de Toulouse, CNRS, UPS, France \\ ${ }^{4}$ Institut de Pharmacologie et Biologie Structurale, Université de Toulouse, CNRS, UPS, France
}

(Received 4 August 2019; published 8 January 2020)

\begin{abstract}
We examine the behavior of supercoiled DNA minicircles containing between 200 and 400 base-pairs, also named microDNA, in which supercoiling favors thermally assisted DNA denaturation bubbles of nanometer size and controls their lifetime. Mesoscopic modeling and accelerated dynamics simulations allow us to overcome the limitations of atomistic simulations encountered in such systems, and offer detailed insight into the thermodynamic and dynamical properties associated with the nucleation and closure mechanisms of long-lived thermally assisted denaturation bubbles which do not stem from bending- or torque-driven stress. Suitable tuning of the degree of supercoiling and size of specifically designed microDNA is observed to lead to the control of opening characteristic times in the millisecond range, and closure characteristic times ranging over well distinct timescales, from microseconds to several minutes. We discuss how our results can be seen as a dynamical bandwidth which might enhance selectivity for specific DNA binding proteins.
\end{abstract}

DOI: 10.1103/PhysRevE.101.012403

\section{INTRODUCTION}

The cooperative opening of a DNA sequence of consecutive base-pairs (bps) is central in biological mechanisms such as replication, transcription, repair, or protein binding [1-4]. From a theoretical point of view, double-strand DNA (dsDNA) segments are in a low-entropy state and carry the enthalpic contributions from the bound bps, whereas the flexible single-strand DNA (ssDNA) denaturation bubbles correspond to entropy reservoirs [5]. Although the DNA macromolecule manifests more thermally driven opening of consecutive bps, also named breathing fluctuations, at physiological temperatures [6], duplex opening can also be at play when nonlinear elastic properties of DNA are involved. This commonly happens when the molecule is strongly bent [7] or negatively supercoiled [8]. Eventually, the duplex opening state becomes stable for sufficient bending or negative superhelical stress [9]. Various experimental [10] and analytical [5,11-15] models have been proposed in the literature to account for the thermodynamic and dynamical properties of denaturation bubbles. However, these approaches did not consider explicitly the twist dynamics, and/or were not able to reach the $100 \mu \mathrm{s}$ experimental timescale, as measured in in vitro experiments [10], of long-lived thermally-assisted denaturation bubbles extending over more than 4 bps. Furthermore, other approaches studied the interplay between denaturation and writhe, but they were limited to non-equilibrium conditions imposed by the dynamic introduction of bending- or torque-driven stress [16-20], which did not give information about equilibrium nucleation and closure rates potentially relevant for fundamental biological processes.

*Corresponding author: francois.sicard@ free.fr
Here, we elucidate the key parameters to obtain longlived thermally assisted bubbles of nanometer size which do not stem from bending- or torque-driven stress in supercoiled DNA minicircles at room temperature. We explore numerically specific design of DNA minicircles containing between 200 and $400 \mathrm{bps}$, also named microDNA, as they are representative of supercoiled DNA loops found in nature [21] and have a suitable size for exploring the relationship between twist and writhe [9]. To overcome the inherent limitations of atomistic simulations encountered at lengthand time-scales of interest [22], mesoscopic modeling [23] is combined with accelerated dynamics simulations $[24,25]$ to study accurately the free energy landscape and the equilibrium rates associated with the nucleation and closure mechanisms of the long-lived thermally-assisted denaturation bubbles. We discuss how specific tuning of DNA structural parameters, such as the minicircle size and degree of supercoiling can lead to a large variety of equilibrium closure and nucleation rates that can be seen as a dynamical bandwidth which might enhance selectivity for DNA binding proteins.

\section{NUMERICAL MODEL}

The dsDNA is described at a mesoscopic scale [23,26], where the two single strands composed of adenine (A), cytosine $(\mathrm{C})$, guanine $(\mathrm{G})$, and thymine $(\mathrm{T})$ are modeled as interacting freely rotating chains of $N$ beads, with each bead representing a nucleotide, as depicted in Fig. 1. These beads interact through two terms: a Morse potential mimicking the inter-strand hydrogen-bonding and an effective intra-strand stacking interaction between the base-pairs modeled through a bare torsional modulus that depends on the distance between complementary bases, $\rho_{i}=\left|\rho_{i}\right|=\left|\mathbf{r}_{i}^{(1)}-\mathbf{r}_{i}^{(2)}\right|$ with $\mathbf{r}_{i}^{(j)}$ the position of bead $i$ on strand $j$, and vanishes for fully separated 


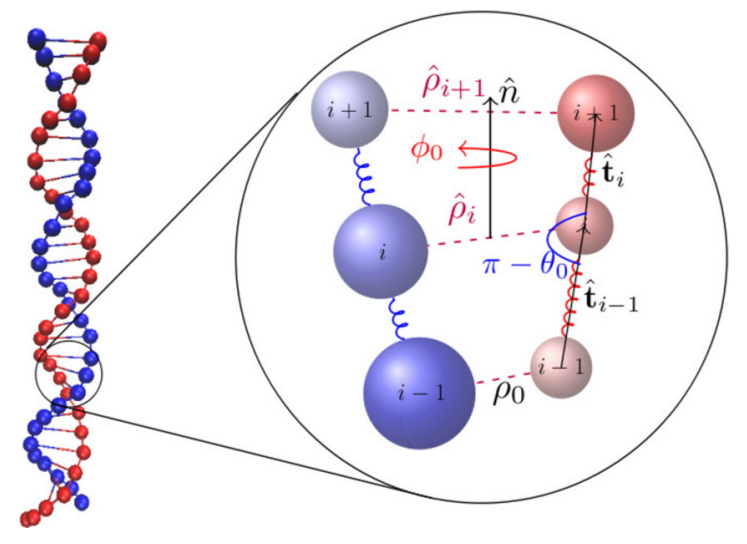

FIG. 1. Snapshot of an equilibrated double helix (from Ref. [26]). The bending angle along each strand is $\theta_{\text {ref }}, \rho_{\text {ref }}$ is the equilibrium base-pair distance, and $\hat{n}$ is the helical axis around which twist is defined. The imposed equilibrium twist between successive pairs is $\phi_{\text {ref. }}$.

strands. The evolution is governed by the overdamped Langevin equation. The full Hamiltonian and the details of the numerical implementation and of the parameter values are given in previous work $[23,26]$ and in Appendix A. For instance, the mesoscopic model yields numerical values for the dsDNA persistence length, $\ell_{\mathrm{ds}} \approx 160 \mathrm{bps}$, and the unconstrained pitch, $p_{0}=12 \mathrm{bps}$, comparable to the actual dsDNA values under physiological conditions [27], in spite of the model simplicity. We focus on a fusible AT-rich region of 30 bps larger than the size of the representative denaturation bubbles studied in this work and clamped by a closed circular GC region [23]. This AT-rich region is flanked by two segments of $10 \mathrm{GC}$ bps on each extremity, which are constrained to be aligned along the $Z$-axis to inhibit the introduction of bendingdriven stress, as depicted in Fig. 2(a). This numerical setup allows us to dissociate, in a first instance, the bending and twist contributions in the nucleation and closure mechanisms of the long-lived thermally assisted denaturation bubble, favoring therefore the convergence of the free energy simulations in the accelerated dynamics framework. As we shall see, the corrections to the numerical estimation of the free energy barrier associated with the closure of the denaturation bubbles are an order of magnitude smaller.

We examine one linear dsDNA ( $\ell D N A$ ) of $N=50 \mathrm{bps}$ and four circular dsDNA (cDNA) with similar AT-rich regions but different lengths $N$ (in bp units) and superhelical densities [28], $\sigma=\frac{L k-L k^{0}}{L k^{0}}=\frac{\Delta L k}{L k^{0}}$, where $L k$ represent the linking numbers of the cDNA molecule [28], i.e., the number of times one backbone strand links through the circle formed by the other, and $L k^{0}$ is defined as $L k^{0}=N / p_{0}$, for any DNA molecule, with $p_{0}=12.0$ (in bp units in the following) the equilibrium pitch measured in the linear state. For a given molecule, the superhelical stress is accommodated by changes in helical twist, $\Delta T w$, and writhe, $\Delta W r$, following $\Delta L k=$ $\Delta T w+\Delta W r$ [16]. As shown in Table I, we consider different values for $\sigma \in[-0.04 ; 0]$. For instance, natural circular DNA molecules, such as bacterial plasmids, vary widely in size, but, when isolated in vitro, the majority have values for $\sigma \leqslant-0.03$ [29]. In the following, the superhelical densities, along with the sizes of the minicircles, are specifically chosen to tune the value of $\Delta L k$. Such specific design allows us to control the interplay between twist and writhe during the formation of the long-lived thermally assisted denaturation bubbles.

Accelerating dynamics simulations are performed within the well-tempered variant of the metadynamics (metaD) framework [30]. This numerical method enhances the sampling of the conformational space of a system along a few selected degrees of freedom, named collective variables (CVs), and reconstructs the equilibrium probability distribution, and thus the free-energy landscape, as a function of these CVs (see details in Appendix B). Here, we considered the width $\rho_{\max }(t)$ of the denaturation bubble, i.e., the maximal distance between paired bases, as $\mathrm{CV}$ to bias the dynamics. We also choose to follow the evolution of the minimal twist angle inside the bubble, $\phi_{\min }(t)=\min _{i \in \text { bubble }} \phi_{i}(t)$, where $\phi_{i} \equiv \arccos \left(\frac{\rho_{i} \cdot \rho_{i+1}}{\rho_{i} \rho_{i+1}}\right)$ is defined as the angle between two consecutive base-pair vectors $\rho_{i}$ and $\rho_{i+1}$, depicted in Fig. 1.

\section{RESULTS AND DISCUSSION}

In Fig. 2(c) are shown the free energy profiles, $\mathcal{F}$, associated with the nucleation and closure mechanisms of the

TABLE I. Linear and circular DNA thermodynamic and dynamical properties. $N / \ell_{\mathrm{ds}}, \sigma$, and $\Delta L k$ correspond to the length, superhelical density and excess linking number of the molecules, respectively, and $\ell_{\mathrm{ds}}=160 \mathrm{bps} . \Delta F_{0}, \Delta F_{\mathrm{op}}$, and $\Delta F_{\mathrm{cl}}$ represent the values of the free energy of formation, opening, and closure, respectively, measured along the minimal free energy paths depicted in Fig. $3 . \Delta F_{0}^{*}$ is the values of the free energy of formation accounting for the entropic contribution in the system. $\tau_{\text {opening }}$ and $\tau_{\text {closure }}$ correspond to the characteristic times for the opening and closure of the long-lived thermally assisted denaturation bubble measured within the metaD framework. The symbol ( $\dagger$ ) means that Eq. (5) was considered to determine the characteristic time.

\begin{tabular}{lccccccccc}
\hline \hline & $N / \ell_{\mathrm{ds}}$ & $\sigma$ & $\Delta L k$ & $\Delta F_{0}\left(k_{B} T\right)$ & $\Delta F_{0}^{*}\left(k_{B} T\right)$ & $\Delta F_{\mathrm{op}}\left(k_{B} T\right)$ & $\Delta F_{\mathrm{cl}}\left(k_{B} T\right)$ & $\tau_{\text {opening }}$ & $\tau_{\text {closure }}$ \\
\hline$\ell \mathrm{DNA}$ & - & - & 0 & $9.0 \pm 0.1$ & $6.7 \pm 0.1$ & $21.8 \pm 0.1$ & $12.9 \pm 0.1$ & $(67 \pm 8) \mathrm{ms}$ & $(121 \pm 12) \mu \mathrm{s}$ \\
$\mathrm{cDNA}_{0}$ & 1.9 & 0 & 0 & $9.9 \pm 0.2$ & $8.1 \pm 0.2$ & $20.9 \pm 0.1$ & $11.1 \pm 0.2$ & $(51 \pm 3) \mathrm{ms}$ & $(17 \pm 2) \mu \mathrm{s}$ \\
$\mathrm{cDNA}_{1 a}$ & 1.5 & -0.008 & -0.17 & $4.3 \pm 0.2$ & $1.2 \pm 0.2$ & $20.5 \pm 0.1$ & $15.9 \pm 0.2$ & $(10.4 \pm 0.6) \mathrm{ms}$ & $(1.7 \pm 0.3) \mathrm{ms}$ \\
$\mathrm{cDNA}_{1 b}$ & 2.3 & -0.008 & -0.25 & $6.5 \pm 0.2$ & $3.4 \pm 0.2$ & $21.0 \pm 0.1$ & $14.6 \pm 0.2$ & $(16.5 \pm 0.7) \mathrm{ms}$ & $(0.33 \pm 0.02) \mathrm{ms}$ \\
$\mathrm{cDNA}_{2 a}$ & 1.5 & -0.024 & -0.5 & $-4.2 \pm 0.2$ & $-8.5 \pm 0.2$ & $19.7 \pm 0.2$ & $23.8 \pm 0.3$ & $(4.9 \pm 0.6) \mathrm{ms}$ & $(26 \pm 17) \mathrm{s} \dagger$ \\
$\mathrm{cDNA}_{2 b}$ & 2.3 & -0.024 & -0.75 & $-0.4 \pm 0.2$ & $-4.2 \pm 0.4$ & $21.6 \pm 0.1$ & $21.7 \pm 0.3$ & $(5.9 \pm 0.5) \mathrm{ms}$ & $(1.1 \pm 0.5) \mathrm{s} \dagger$ \\
$\mathrm{cDNA}_{3 a}$ & 1.4 & -0.04 & -0.75 & $-5.0 \pm 0.4$ & $-9.4 \pm 0.4$ & $21.8 \pm 0.1$ & $26.8 \pm 0.5$ & $(7.2 \pm 0.6) \mathrm{ms}$ & $(3.7 \pm 2.6) \mathrm{min} \dagger$ \\
$\mathrm{cDNA}_{3 b}$ & 1.6 & -0.04 & -0.83 & $-4.4 \pm 0.4$ & $-9.4 \pm 0.3$ & $20.9 \pm 0.3$ & $25.4 \pm 0.7$ & $(14.2 \pm 1.0) \mathrm{ms}$ & $(1.8 \pm 1.1) \mathrm{min} \dagger$ \\
\hline \hline
\end{tabular}



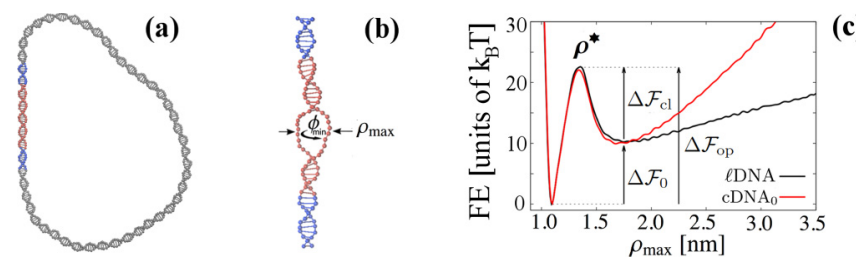

FIG. 2. Equilibrium snapshots of (a) circular DNA with pitch $p=12.0$ base-pairs ( $\left.\mathrm{cDNA}_{0}\right)$ and (b) linear dsDNA ( $\left.\ell \mathrm{DNA}\right)$ when the long-lived denaturation bubble is formed. The AT-rich region of size 30 base-pairs (red) is delimited at each extremity by two sequences of $10 \mathrm{GC}$ bps aligned arbitrarily along the $Z$-axis (blue). $\mathrm{cDNA}_{0}$ is closed by a circular GC region (grey). The maximal distance between paired bases, $\rho_{\max }$, and the minimal twist angle between successive bps, $\phi_{\min }$, defined in the main text are shown. (c) Free energy profiles associated with the opening and closure mechanism of $\ell$ DNA and $\mathrm{cDNA}_{0}$ projected along $\rho_{\max }$. The location of the transition state, $\rho^{\star}$, is shown.

long-lived thermally assisted denaturation bubble in the linear and circular dsDNA with $\sigma=0$, obtained within the metaD framework and projected along the width $\rho_{\max }$ of the bubble depicted in Fig. 2(b). In both systems, a closure free energy barrier, $\Delta \mathcal{F}_{\mathrm{cl}} \approx 12.3 k_{B} T$ (with $T=300 \mathrm{~K}$ is room temperature) separates the metastable basins associated with the denaturation bubble $\left(\rho_{\max } \geqslant 1.35 \mathrm{~nm}\right)$ from the closed state basin $\left(\rho_{\max } \approx 1.1 \mathrm{~nm}\right)$. These two basins are well separated by a free energy of formation $\Delta \mathcal{F}_{0} \approx 10.3 k_{B} T$, defining the opening free energy barrier, $\Delta \mathcal{F}_{\mathrm{op}} \equiv \Delta \mathcal{F}_{0}+\Delta \mathcal{F}_{\mathrm{cl}} \approx$ $22.6 k_{B} T$, associated with the nucleation mechanism. These values can be compared with previous work [23], where the formation of denaturation bubble in linear dsDNA without alignment constraint on the GC segments flanking the AT-rich region was studied. We measured in [23] a very similar value for $\Delta \mathcal{F}_{\text {op }}$, but a free energy difference of $\approx 2 k_{B} T$ in $\Delta \mathcal{F}_{0} \approx$ $8 k_{B} T$ and $\Delta \mathcal{F}_{\mathrm{cl}} \approx 14 k_{B} T$. This difference in the free energies is about the thermal fluctuation scale and represents the loss of configurational entropy associated with the alignment of the $\mathrm{GC}$ regions during the closure of the AT-rich region.

The molecules $\mathrm{cDNA}_{0}$ and $\ell \mathrm{DNA}$ only differ from each other by their boundary conditions with or without the closure of the GC regions located on each side of the AT-rich region. It yields the reduction of the configurational entropy contribution of the system in the metastable basin associated with the long-lived thermally assisted denaturation bubble. This is qualitatively shown in Fig. 3 where the free energy surfaces (FES) are reconstructed within the metaD framework along the two CVs, $\rho_{\max }$ and $\phi_{\min }$. The entropic contribution to the FES can be quantitatively assessed considering the definition of the free energy difference in terms of the joint probability distribution of the CVs [25,31],

$$
\Delta F_{0}^{*}=-k_{B} T \log \left(\frac{P_{\mathrm{op}}}{P_{\mathrm{cl}}}\right) .
$$

In Eq. (1), $P_{\mathrm{cl}}$ and $P_{\mathrm{op}}$ are the probabilities of the closed and open DNA states, respectively. The probability of each state is computed as the integral of the distribution within the energy basin, $\mathcal{B}$, it occupies in the $\mathrm{CV}$-space,

$$
P_{i}=\iint_{\left(\rho_{\max }, \phi_{\min }\right) \in \mathcal{B}_{\mathrm{i}}} f\left(\rho_{\max }, \phi_{\min }\right) d \rho_{\max } d \phi_{\min },
$$

where $f$ is the joint probability density distribution function computed within the metaD framework associated with the system free energy. We considered the successive isosurfaces depicted in Fig. 3 as integration domains. We report in Table I the value of the free energy of formation between the two basins observed in Fig. 3, $\Delta F_{0}^{*}$, computed with Eq. (1). As we could expect from visual inspection in Figs. 2(c) and 3, the free energy landscapes show significant differences between $\mathrm{cDNA}_{0}$ and $\ell \mathrm{DNA}$ associated with the difference in the global entropic contribution in the free energy basins.

In Fig. 3 is also shown the evolution of the FES, reconstructed within the metaD framework along $\rho_{\max }$ and $\phi_{\min }$, when the superhelical density of the system goes from $\sigma=0$ to -0.04 . As we would expect from energetic consideration [8], the change of sign of both the free energy of formation measured along the minimal free energy paths (MFEPs) depicted in Fig. 3, $\Delta F_{0}$, and the free energy of formation evaluated as a function of the joint probability distribution defined in Eq. (1), $\Delta F_{0}^{*}$, in Table I shows the progressive inversion of the thermodynamic stability of the system for increasing undertwist. This is characteristic of the predominant stability of the long-lived thermally assisted denaturation bubble. This transition comes with the drift of the location of the nucleation basin towards larger values of $\rho_{\max }$, which is representative of the increase of the size of the denaturation bubble (see Table II). As reported in Table I, the impact of the superhelical density, $\sigma$, on the denaturation bubble stability is also shown
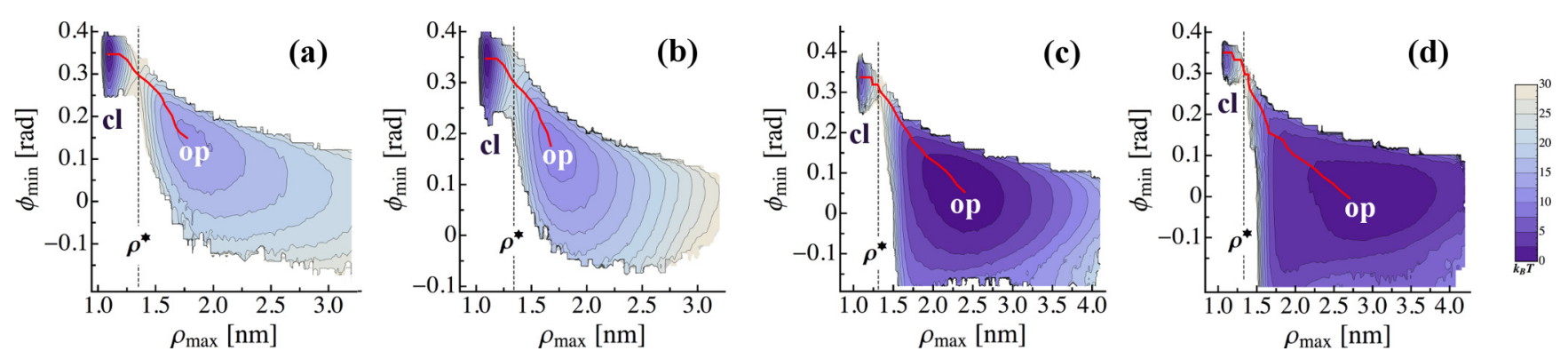

FIG. 3. Free energy surface of $\ell$ DNA (a), $\operatorname{cDNA}_{0}(\mathrm{~b}), \mathrm{cDNA}_{2 a}$ (c), and $\mathrm{cDNA}_{3 a}$ (d) projected along the maximal distance between paired bases, $\rho_{\max }$, and the minimal twist angle between successive bps, $\phi_{\min }$, defined in the main text in the linear and circular DNAs reported in Table I. The free energy basins associated with the open (op) and closed (cl) states of the DNA bubble, the location of the transition state $\left(\rho^{\star}\right)$, and the typical minimal free energy paths obtained within the steepest descent framework (red) are shown. 
TABLE II. Configurational features of the long-lived denaturation bubble in the linear and circular dsDNAs. $\rho_{\max }^{\text {bub }}, \phi_{\min }^{\text {bub }}$ and $N_{\mathrm{av}}^{\text {bub }}$ $\left(N_{\max }^{\mathrm{bub}}\right)$ correspond to the position of the nucleation free-energy basin in Fig. 3, and the average (maximal) number of opened base-pairs in the denaturation bubble, delimited within $2 k_{B} T$ from the free energy minimum.

\begin{tabular}{lcrr}
\hline \hline & $\rho_{\text {max }}^{\text {bub }}(\mathrm{nm})$ & $\phi_{\text {min }}^{\text {bub }}(\mathrm{rad})$ & \multicolumn{1}{c}{$N_{\mathrm{av}}^{\text {bub }}\left(N_{\mathrm{max}}^{\text {bub }}\right)$} \\
\hline$\ell \mathrm{DNA}$ & $1.8 \pm 0.2$ & $0.15 \pm 0.05$ & $9 \pm 3(16)$ \\
$\mathrm{cDNA}_{0}$ & $1.7 \pm 0.1$ & $0.17 \pm 0.05$ & $8 \pm 2(14)$ \\
$\mathrm{CDNA}_{1 a}$ & $1.8 \pm 0.1$ & $0.15 \pm 0.04$ & $8 \pm 2(16)$ \\
$\mathrm{CDNA}_{1 b}$ & $1.8 \pm 0.2$ & $0.15 \pm 0.05$ & $9 \pm 2(18)$ \\
$\mathrm{cDNA}_{2 a}$ & $2.4 \pm 0.2$ & $0.06 \pm 0.05$ & $12 \pm 2(20)$ \\
$\mathrm{CDNA}_{2 b}$ & $2.3 \pm 0.5$ & $0.08 \pm 0.04$ & $12 \pm 3(22)$ \\
$\mathrm{CDNA}_{3 a}$ & $3.0 \pm 0.5$ & $-0.01 \pm 0.06$ & $14 \pm 3(22)$ \\
$\mathrm{cDNA}_{3 b}$ & $3.1 \pm 0.5$ & $-0.02 \pm 0.06$ & $15 \pm 3(26)$ \\
\hline \hline
\end{tabular}

with the increase of the closure free energy measured along the MFEPs, $\Delta F_{\mathrm{cl}}$, which is maximal when $\sigma=-0.04$.

Interestingly, the results reported in Table I show that, in the absence of bending or torque-driven stress, the opening free energy measured along the MFEPs, $\Delta F_{\mathrm{op}}$, does not significantly depend on the value of the superhelical density, $\sigma$. They suggest, however, that the response of the cDNAs depends on $N / \ell_{\mathrm{ds}}$, itself related to the flexibility of the dsDNAs. This behavior is in line with the work of Sayar et al. [28] where the fraction of the linking number absorbed as twist and writhe was studied when circular DNAs of different lengths approach the supercoiling transition. For dsDNA chains of the order of one persistence length, and $\Delta L k<$ 1 , the authors showed that the excess linking number was essentially absorbed by the change in twist. For longer chains with $N / \ell_{\mathrm{ds}}>2$ (i.e., longer than Kuhn's length in the dsDNA state), instead, they observed an increasing fraction of the linking number absorbed by the writhe (see Table III). Indeed in this case the bending energy cost induced by the writhe is smaller. In our cDNAs (see Table I) this nontrivial dependence on chain length and excess linking number is reflected in the corresponding adjustment in the free energy of formation, $\Delta F_{0}^{*}$, and the closure free energy measured along the MFEPs, $\Delta F_{\mathrm{cl}}$.

More sophisticated approach would necessarily take into account the entropy brought by the relative misalignment of the sequences on both sides of the AT-rich region, that we have so far ignored, notably playing a role during the initiation stage of the denaturation bubble closure $[23,26]$. In the topologically unconstrained case, the bending contribution can be assessed by modeling the denaturation bubble as a single rotating joint, as the typical bubble length $(\sim 10 \mathrm{bps})$ is on the order of the ssDNA Kuhn's length, $2 \ell_{p}^{\text {ss }} \simeq 8 \mathrm{~nm}$. Denoting $\kappa$ the joint bending rigidity, the denaturation bubble's state can be characterized by the angle $\theta$ and elastic energy $\kappa(1-\cos \theta)$. The associated partition function is

$$
Z_{\kappa}=\int_{0}^{\pi} \mathrm{d} \theta 2 \pi \sin \theta e^{-\beta \kappa(1-\cos \theta)}=4 \pi e^{-\beta \kappa} \frac{\sinh \beta \kappa}{\beta \kappa} .
$$

As compared to the constrained case $(\mathbf{C})$ where the arms are forced to be aligned, the free energy difference in the unconstrained case $(\mathbf{U})$ is $\Delta F_{\kappa}=F_{\kappa}^{\mathbf{C}}-F_{\kappa}^{\mathbf{U}}=k_{\mathrm{B}} T \ln Z_{\kappa}>0$. The value of $\kappa$ in the present case is difficult to evaluate because the joint is composed of several base-pairs. It can be estimated as $\kappa \approx 2 k_{\mathrm{B}} T \ell_{p}^{\mathrm{ss}} / N_{\text {bub }}$ because there are two strands in the bubble of length $N_{\text {bub }} \approx 10$ bps. One obtains $\beta \kappa \approx 2$ and $\Delta F_{\kappa} \simeq 1.2 k_{\mathrm{B}} T$. In any case, whatever the value of $\kappa$, the free energy gain due to arm alignment is lower than $\lim _{\kappa \rightarrow 0} \Delta F_{\kappa}=k_{\mathrm{B}} T \ln 4 \pi \simeq 2.5 k_{\mathrm{B}} T$, in agreement with the numerical comparison between $\ell$ DNA or $\mathrm{CDNA}_{0}$ and the linear DNA without alignment constraint studied in Ref. [23].

To go further, we additionally measured the difference in torsional energy in the open state between the constrained system $(\mathbf{C})$ and the unconstrained system $(\mathbf{U})$, as obtained in unbiased numerical simulations. In this optic, we considered the circular DNA with superhelical density $\sigma=0\left(\mathrm{cDNA}_{0}\right)$ and $-0.04\left(\mathrm{cDNA}_{3 b}\right)$ and measured the torsional energy stored in the dsDNA state delimiting the AT-rich region

$$
\mathcal{H}_{\mathrm{tor}}^{\mathrm{dsDNA}}=\sum_{i \in \mathrm{dsDNA}} \frac{\kappa_{\phi}}{2}\left(\phi_{i}-\phi_{\mathrm{ref}}\right)^{2},
$$

where $\phi_{i}$ is defined as the angle between two consecutive base-pair vectors, $\phi_{\text {ref }}=0.547 \mathrm{rad}$ (see Table III) and $\kappa_{\phi}=$ $450 k_{B} T$ is the value of the torsional modulus obtained after equilibration (see Ref. [23]). We obtained a difference in torsional energy between the constrained and unconstrained configurations $\Delta F_{\text {tor }}=F_{\text {tor }}^{\mathbf{C}}-F_{\text {tor }}^{\mathbf{U}} \approx 0.5 k_{B} T$ and $1.5 k_{B} T$ for $\mathrm{cDNA}_{0}$ and $\mathrm{cDNA}_{3 b}$, respectively, ignoring rotational entropy. We then estimate the correction in free energy between the constrained and unconstrained configurations on the closure of the denaturation bubble, $\Delta F_{\text {corr }}=F_{\text {corr }}^{\mathbf{C}}-F_{\text {corr }}^{\mathbf{U}}=\Delta F_{\kappa}+$

TABLE III. Linear ( $\ell$ DNA) and circular (cDNA) dsDNA parameters for the set of initial configurations of sizes, $N$, and difference in linking number, $\Delta L k$, considered throughout this study. The theoretical (th) and numerical (num) values obtained for the equilibrated dsDNA

\begin{tabular}{|c|c|c|c|c|c|c|c|c|}
\hline & $p^{(\mathrm{th})}(\mathrm{bps})$ & $N$ (bps) & $N / \ell_{\mathrm{ds}}$ & $\Delta L k$ & $\phi_{\mathrm{eq}}^{(\mathrm{th})}(\mathrm{rad})$ & $\phi_{\mathrm{eq}}^{(\mathrm{num})}(\mathrm{rad})$ & $W r_{\mathrm{eq}}^{(\text {num })}$ & $\sigma$ \\
\hline$\ell \mathrm{DNA}$ & 12.0 & 50 & - & 0 & 0.524 & $0.547 \pm 0.047$ & - & - \\
\hline $\mathrm{cDNA}_{0}$ & 12.0 & 300 & 1.9 & 0 & 0.524 & $0.528 \pm 0.050$ & $0.02 \pm 0.03$ & 0 \\
\hline $\mathrm{cDNA}_{1 a}$ & 12.1 & 242 & 1.5 & -0.17 & 0.519 & $0.527 \pm 0.051$ & $-0.09 \pm 0.06$ & -0.008 \\
\hline $\mathrm{cDNA}_{1 b}$ & 12.1 & 363 & 2.3 & -0.25 & 0.519 & $0.527 \pm 0.051$ & $-0.17 \pm 0.06$ & -0.008 \\
\hline $\mathrm{cDNA}_{2 a}$ & 12.3 & 246 & 1.5 & -0.5 & 0.511 & $0.520 \pm 0.051$ & $-0.15 \pm 0.04$ & -0.024 \\
\hline $\mathrm{cDNA}_{2 b}$ & 12.3 & 369 & 2.3 & -0.75 & 0.511 & $0.523 \pm 0.052$ & $-0.38 \pm 0.05$ & -0.024 \\
\hline $\mathrm{cDNA}_{3 a}$ & 12.5 & 225 & 1.4 & -0.75 & 0.503 & $0.519 \pm 0.052$ & $-0.37 \pm 0.06$ & -0.04 \\
\hline $\mathrm{cDNA}_{3 b}$ & 12.5 & 250 & 1.6 & -0.83 & 0.503 & $0.524 \pm 0.051$ & $-0.61 \pm 0.08$ & -0.04 \\
\hline
\end{tabular}
are given for the pitch, $p$, twist angle, $\phi$, writhe, $W r$, and superhelical density, $\sigma$. 
TABLE IV. Linear ( $\ell$ DNA) and circular (cDNA) dsDNA parameters derived from the accelerated dynamics simulations. The parameters $\left(\operatorname{det} \mathbf{H}_{\mathrm{op}}\right)^{1 / 2}$ and $\left(\operatorname{det} \mathbf{H}_{\mathrm{cl}}\right)^{1 / 2}$ represent the effective stiffness of the free energy well associated with the open (op) and closed (cl) dsDNA states, respectively, as depicted in Fig. $3 . k_{\text {opening }} / k_{\text {closure }}$ is the ratio of the transition rates associated with the opening (cl $\rightarrow$ op) and closure (op $\rightarrow \mathrm{cl}$ ) of the long-lived thermally assisted DNA denaturation bubble. The characteristic times derived from the accelerated dynamics simulations are given along with the respective $p$-value obtained from the Kolmogorov-Smirnov test. The symbol (-) indicates that Eq. (C4) was considered to determine the characteristic time.

\begin{tabular}{|c|c|c|c|c|c|c|c|}
\hline & $\left(\operatorname{det} \mathbf{H}_{\mathrm{op}}\right)^{1 / 2}$ & $\left(\operatorname{det} \mathbf{H}_{\mathrm{cl}}\right)^{1 / 2}$ & $k_{\text {opening }} / k_{\text {closure }}$ & $\tau_{\text {opening }}$ & $p$-value & $\tau_{\text {closure }}$ & $p$-value \\
\hline$\ell D N A$ & $441 \pm 25$ & $(1.1 \pm 0.3) \times 10^{-4}$ & $(3.1 \pm 1.3) \times 10^{-3}$ & $(67 \pm 8) \mathrm{ms}$ & 0.65 & $(121 \pm 12) \mu \mathrm{s}$ & 0.86 \\
\hline $\mathrm{cDNA}_{0}$ & $750 \pm 57$ & $(1.3 \pm 0.2) \times 10^{-4}$ & $(8.4 \pm 3.6) \times 10^{-4}$ & $(51 \pm 3) \mathrm{ms}$ & 0.80 & $(17 \pm 2) \mu \mathrm{s}$ & 0.52 \\
\hline $\mathrm{cDNA}_{1 a}$ & $471 \pm 41$ & $(1.1 \pm 0.3) \times 10^{-4}$ & $(3.2 \pm 1.8) \times 10^{-1}$ & $(10.4 \pm 0.6) \mathrm{ms}$ & 0.98 & $(1.7 \pm 0.3) \mathrm{ms}$ & 0.62 \\
\hline $\mathrm{cDNA}_{1 b}$ & $457 \pm 12$ & $(1.2 \pm 0.4) \times 10^{-4}$ & $(3.8 \pm 2.0) \times 10^{-2}$ & $(16.5 \pm 0.7) \mathrm{ms}$ & 0.82 & $(0.33 \pm 0.02) \mathrm{ms}$ & 0.79 \\
\hline $\mathrm{cDNA}_{2 a}$ & $142 \pm 15$ & $(1.2 \pm 0.3) \times 10^{-4}$ & $(5.2 \pm 2.9) \times 10^{3}$ & $(4.9 \pm 0.6) \mathrm{ms}$ & 0.71 & $(26 \pm 17) \mathrm{s}$ & - \\
\hline $\mathrm{cDNA}_{2 b}$ & $101 \pm 3$ & $(1.3 \pm 0.2) \times 10^{-4}$ & $(1.8 \pm 0.7) \times 10^{2}$ & $(5.9 \pm 0.5) \mathrm{ms}$ & 0.66 & $(1.1 \pm 0.5) \mathrm{s}$ & - \\
\hline $\mathrm{cDNA}_{3 a}$ & $75 \pm 4$ & $(1.2 \pm 0.2) \times 10^{-4}$ & $(3.1 \pm 1.9) \times 10^{4}$ & $(7.2 \pm 0.6) \mathrm{ms}$ & 0.71 & $(3.7 \pm 2.6) \mathrm{min}$ & - \\
\hline $\mathrm{cDNA}_{3 b}$ & $130 \pm 1$ & $(1.2 \pm 0.2) \times 10^{-4}$ & $(7.4 \pm 4.2) \times 10^{3}$ & $(14.2 \pm 1.0) \mathrm{ms}$ & 0.93 & $(1.8 \pm 1.1) \min$ & - \\
\hline
\end{tabular}

$\Delta F_{\text {tor }} \approx 1.7 k_{B} T$ and $2.7 k_{B} T$ for $\mathrm{cDNA}_{0}$ and $\mathrm{cDNA}_{3 b}$, respectively.

Finally, building on accelerated dynamics frameworks [24,32] and transition state theory [33,34], we assessed numerically the characteristic times $\tau_{\text {opening }}$ and $\tau_{\text {closure }}$ associated with the opening and closure of the long-lived thermally assisted denaturation bubbles, respectively, when the arms on each extremity of the AT-rich region are forced to be aligned. To take into account the presence of slow nonreactive modes, as characterized by the configurational entropic contribution, the slow mode dynamics must be treated explicitly on an equal footing with the mode along the reaction coordinate. This issue can be addressed within the multidimensional Kramers-Langer's (KL) framework [35-38], which yields the expression for the transition rate

$$
k_{K L}=\frac{1}{2 \pi}\left(\frac{\operatorname{det} \mathbf{H}\left(q_{0}\right)}{\left|\operatorname{det} \mathbf{H}\left(q_{T}\right)\right|}\right)^{1 / 2} \lambda_{T} e^{-\Delta F / k_{B} T} .
$$

In Eq. (5), $\mathbf{H}\left(q_{0}\right)$ and $\mathbf{H}\left(q_{T}\right)$ are the Hessian matrices of the free energy function with respect to coordinates at the well bottom and the transition state, respectively, $\mathbf{D}$ is the diffusion tensor, and $\lambda_{T}$ is the only positive root of the equation $\operatorname{det}(\lambda \mathbf{I}+\mathbf{H D})=0$ defined in the transition region [35-37] (see details in Appendix C). The characteristic times can thus be defined as the inverse of the transition rate, $k_{K L}$, defined in Eq. (5). The results reported in Table I show a broad range of characteristic times associated with either the opening or the closure of the denaturation bubble of nanometer size. For instance, the characteristic opening time and equilibrium constant obtained from our study in the case of the linear dsDNA ( $\ell D N A)$ are in good agreement with previous work [23] and the experimental results of Englander et al. [39] and more recently Altan-Bonnet et al. [10], where an Arrhenius-like exponential dependence of the mean transition times has been measured. As qualitatively shown in Fig. 3 and quantitatively assessed in Table IV, the results reported in Table I show equilibrium times, which depend on the interplay between energetic and entropic characteristics of the undertwisted circular DNAs. For instance, we observed opening times in the millisecond range, which are relatively unstressed by different degree of supercoiling. However, configurational entropy associated with the torsional constraint induced by similar $\sigma$ but different $\Delta L k$ can significantly influence the closure times over several orders of magnitude. Taking into account the misalignment of the sequences on both sides of the AT-rich region during the denaturation bubble closure, as discussed in the thermodynamic context above [see Eqs. (3) and (4)], we estimate a correction factor between the constrained and unconstrained configurations in the closure characteristic times reported in Table I of between 5 and 15 depending on the superhelical density of the system.

\section{CONCLUSION}

The extensive simulations discussed above allowed us to decipher the thermodynamic and dynamical characteristics of long-lived thermally assisted denaturation bubbles of nanometer size which do not stem from bending- or torque-driven stress in undertwisted microDNA containing between 200 and $400 \mathrm{bps}$. Even though the numerical values derived above could be approximate because of our coarse-grained model, our results show that suitable tuning of the degree of supercoiling and size of specifically designed microDNA would allow the control of opening and closure characteristic times, ranging over well distinct timescales, from microseconds to several minutes. Interestingly, we showed that these dynamical characteristics can be related to specific tuning of both energetic and entropic properties of the DNA minicircles. To go further, it would be interesting to take into account in the calculation leading to Eq. (3) the role of bending constraints induced by the topology of the supercoiled DNA minicircle on the base-pair segments delimiting both sides of the fusible AT-rich region. In this situation, a coupling between bending (writhe) and twist would be likely to occur, which would impact the thermodynamic and dynamical properties associated with the nucleation and closure mechanisms of long-lived thermally assisted DNA denaturation bubbles. This road map will be considered in the near future.

Finally, let us comment on the potential biological implications of this work. As DNA supercoiling is determinant in the stability of the long-lived thermally assisted DNA denaturation bubbles, the DNA minicircle could be used as a direct transducer of supercoiling induced by protein-binding 
into denaturation bubbles with controlled lifetimes. For example, the bacteriophage $\lambda \mathrm{O}$ protein and gal Repressor, which are well-characterized DNA replication initiator and E. coli transcription factors, were shown to induce excess linking numbers $\Delta L k=-0.222 \pm 0.016$ and $\Delta L k=-0.169 \pm$ 0.004 on plasmid of about $4.8 \mathrm{kbps}$ containing 16 and 18 sites, respectively [40]. We predict that the binding of such proteins on DNA molecules containing between 200 and 400 base-pairs, as studied here, would induce long-lived thermally assisted DNA denaturation bubbles with characteristic times $\tau_{\text {closure }}$ in the order of hundreds of nanoseconds for the $\lambda \mathrm{O}$ protein (respectively the millisecond for the gal Repressor) and $\tau_{\text {opening }}$ in the order of tens of milliseconds, which could be measured by smFRET on freely diffusing DNA [41].

Otherwise, the broad range of closure and nucleation times that we predicted in this work could offer a dynamical bandwidth for a breathing DNA binding sensor. The large set of minicircles studied could thus be employed as sensors for the growing number of nucleoproteins with binding propensity for breathing DNA. The prokaryotic transcription factor Fis is one of them since Alexandrov and coworkers have reported that its binding affinity was enhanced correlatively with the enhanced breathing dynamics of its specific binding sequences [42]. Another one is the human single-stranded DNA binding protein 1 (hSSB1), involved in the repair of DNA damage, which was shown to be recruited to dsDNA breaks within only $10 \mathrm{~s}$ after the breakage event as if hSSB1 had an enhanced sensitivity for breathing DNA [43]. The minicircles employed as breathing DNA binding sensor could therefore permit to unravel the detailed mechanism of hSSB1 binding and its dynamics, and promote the design of new hSSB 1 inhibitors, which would consequently enhance the cell sensitivity to chemo- and radiotherapy and reduce the toxicity of anti-cancer treatments [44].

\section{ACKNOWLEDGMENTS}

We acknowledge L. Salomé and A. K. Dasanna for useful discussions. F.S. thanks J. Cuny and M. Salvalaglio for fruitful discussion concerning the metadynamics framework.

\section{APPENDIX A: NUMERICAL MODEL}

To overcome the inherent limitations of atomistic simulations encountered at length- and time-scales of interest [22], we use the DNA model of Refs. [23,26], where the mesoscopic DNA model consists in two interacting beadspring chains each made of $N$ beads (of diameter $a=0.34$ $\mathrm{nm})$ at position $\mathbf{r}_{i}$, with a AT-rich region of 30 base-pairs (bps) clamped with a GC region of $N-30 \mathrm{bps}$, as shown in Fig. 2(b). The Hamiltonian is $\mathcal{H}=\mathcal{H}_{\mathrm{el}}^{(1)}+\mathcal{H}_{\mathrm{el}}^{(2)}+\mathcal{H}_{\text {tor }}+$ $\mathcal{H}_{\text {int }}$, where the first two contributions are elastic energies of the strands $j=1,2$ which include both stretching and bending energies

$$
\mathcal{H}_{\mathrm{el}}^{(j)}=\sum_{i=0}^{N-1} \frac{\kappa_{s}}{2}\left(r_{i, i+1}-a_{\mathrm{ref}}\right)^{2}+\sum_{i=0}^{N-1} \frac{\kappa_{\theta}}{2}\left(\theta_{i}-\theta_{\mathrm{ref}}\right)^{2} .
$$

The stretching modulus, $a^{2} \beta \kappa_{s}=100$, is a compromise between numerical efficiency and experimental values [45], where $\beta^{-1}=k_{B} T$ is the thermal energy, $T=300 \mathrm{~K}$ is the room temperature, and $a_{\text {ref }}=0.357 \mathrm{~nm}$. The bending modulus is large, $\beta \kappa_{\theta}=600$, to maintain the angle between two consecutive tangent vectors along each strand $\theta_{i}$ to the fixed value $\theta_{\text {ref }}=0.41 \mathrm{rad}$ (see Fig. 1). Each strand is thus modeled as a freely rotating chain (FRC). The third and fourth terms of $\mathcal{H}$ are the torsional energy and hydrogen-bonding interactions, respectively. The torsional energy is modeled by a harmonic potential

$$
\mathcal{H}_{\text {tor }}=\sum_{i=0}^{N-1} \frac{\kappa_{\phi, i}}{2}\left(\phi_{i}-\phi_{\text {ref }}\right)^{2}
$$

where $\phi_{i}$ is defined as the angle between two consecutive base-pair vectors $\boldsymbol{\rho}_{i} \equiv \mathbf{r}_{i}^{(1)}-\mathbf{r}_{i}^{(2)}$ and $\boldsymbol{\rho}_{i+1}\left(\phi_{\mathrm{ref}}=0.62 \mathrm{rad}\right)$. The stacking interaction between base pairs is modeled through a $\kappa_{\phi, i}$ that depends on the value of the bare dsDNA torsional modulus $\kappa_{\phi}$, and the distances between complementary bases, $\kappa_{\phi, i}=\kappa_{\phi}\left[1-f\left(\rho_{i}\right) f\left(\rho_{i+1}\right)\right]$, where

$$
f\left(\rho_{i}\right)=\frac{1}{2}\left[1+\operatorname{erf}\left(\frac{\rho_{i}-\rho_{b}}{\lambda^{\prime}}\right)\right],
$$

and $\rho_{i}=\left|\boldsymbol{\rho}_{i}\right|$. Hence, $\kappa_{\phi, i}=\kappa_{\phi}$ in the dsDNA state and $\kappa_{\phi, i}=$ 0 in the ssDNA one. Considering preliminary works $[23,26]$, we have chosen $\lambda^{\prime}=0.15 \mathrm{~nm}, \rho_{b}=1.20 \mathrm{~nm}$, and $\beta \kappa_{\phi}=540$, which yields thermodynamic and dynamical properties in good agreement with biophysical mechanisms. The hydrogenbonding interaction is modeled by a Morse potential

$$
\mathcal{H}_{\text {int }}=\sum_{i=0}^{N-1} A\left(e^{-2 \frac{\rho_{i}-\rho_{\mathrm{ref}}}{\lambda}}-2 e^{-\frac{\rho_{i}-\rho_{\mathrm{ref}}}{\lambda}}\right),
$$

where $\rho_{\text {ref }}=1 \mathrm{~nm}, \lambda=0.2 \mathrm{~nm}$, and $\beta A=8$ and 12 for AT and GC bonding, respectively, as in Refs. [46,26,23]. The fitted values for the dsDNA persistence length and the pitch are $\ell_{\mathrm{ds}} \simeq 160 \mathrm{bps}$ and $p=12$ bps for the relevant range of $\beta \kappa_{\phi}$ we are interested in, which are comparable to the actual dsDNA values $\left(\ell_{\mathrm{ds}} \simeq 150 \mathrm{bps}\right.$ and $\left.p=10.4 \mathrm{bps}\right)$. The ssDNA persistence length is $\ell_{\mathrm{ss}}=3.7 \mathrm{~nm}$, compatible with experimental measurement [47], even though in the upper range of measured values.

We restrain our analysis to four different circular dsDNAs (cDNA) with different superhelical density, $\sigma$, but with a similar sequence of bps. As shown in Table III, the reference pitchs, $p^{\text {(th) }}$, of $\mathrm{cDNA}_{0}, \mathrm{cDNA}_{1}, \mathrm{cDNA}_{2}$, and $\mathrm{cDNA}_{3}$ are initially set to $p^{\text {(th) }}=12.0,12.1,12.3$, and $12.5 \mathrm{bps}$, respectively. The number of beads on each strand, $N$, is chosen so that the number of axis segment, $N / p$, be an integer, and $\ell_{\mathrm{ds}}<N<$ $400 \mathrm{bps}$, as it is representative of the supercoiled DNA loops found in nature [21,48-51]. The superhelical densities, along with the sizes $N$ of the minicircles, were specifically chosen to tune the value of $\Delta L k<1$. Such specific design allowed us to control the interplay between twist and writhe during the formation of the long-lived thermally assisted denaturation bubbles [28]. Furthermore, to quantify the role of the boundary conditions on the formation of the denaturation bubble, we considered a linear dsDNA of $N=50$ bps made of a similar AT-rich region of $30 \mathrm{bps}$ flanked by two segments of $10 \mathrm{GC}$ bps on each extremity ( $\ell D N A)$. To allow the comparison of the degree of supercoiling between molecules of different 
sizes, we normalize the measurement of the supercoiling with the use of the superhelical density $[28,52]$

$$
\sigma=\frac{L k-L k^{0}}{L k^{0}}=\frac{\Delta L k}{L k^{0}},
$$

where $L k$ represents the linking numbers of the cDNA molecule [28,52], and $L k^{0}$ is defined as $L k^{0}=N / p_{0}$ for any DNA molecule with $p_{0}=12.0 \mathrm{bps}$ the equilibrium pitch measured in the linear state. $L k$ is a topological property of circular DNA that does not depend on its particular conformation $[8,53]$, and obeys the relation

$$
L k=T w+W r,
$$

where $T w$ represents the helical twist (the number of times either backbone winds around the helix axis), and $W r$ represents the writhe, or degree of supercoiling (the number of signed crossing of the helix axis in planar projection, averaged over all projection directions). Although $L k$ is a topological invariant integer, $W r$ and $T w$ are not and depend on the geometry [54]. For a given molecule, the superhelical stress produced by deviations of $L k$ from $L k^{0}$ is accommodated by changes in $T w, W r$, or both, following

$$
\Delta L k=\left(L k-L k^{0}\right)=\Delta T w+\Delta W r .
$$

Here, $\Delta T w$ corresponds to localized, sequence-dependent twist deformations such as strand separation or double-helical structure transitions. $\Delta W r$ corresponds to bent (supercoiling) deformations [54].

\section{APPENDIX B: MD SIMULATION}

The evolution of the system is governed by Brownian dynamics, i.e., simulations based upon numerical integration of the overdamped Langevin equation [16,23,26,55], integrated using a Euler's scheme,

$$
\zeta \frac{d \mathbf{r}_{i}}{d t}=-\nabla_{\mathbf{r}_{i}} \mathcal{H}\left(\mathbf{r}_{j}\right)+\xi(t)
$$

where $\zeta=3 \pi \eta a$ is the friction coefficient for each bead of diameter $a$ with $\eta=10^{-3} \mathrm{~Pa}$ s the water viscosity. The diffusion coefficient, $D_{\text {diff }} \equiv k_{B} T / 3 \pi \eta a$, thus takes into account the level of coarse-graining of the mesoscopic model involved in the kinetics associated with the smoothed free energy landscape [56]. The random force of zero mean $\xi_{i}(t)$ obeys the fluctuation-dissipation relation $\left\langle\xi_{i}(t) . \xi_{i}\left(t^{\prime}\right)\right\rangle=6 k_{B} T \zeta \delta_{i j} \delta(t-$ $\left.t^{\prime}\right)$. Lengths and energies are made dimensionless in the units of $a=0.34 \mathrm{~nm}$ and $k_{B} T$, respectively. The dimensionless time step is $\delta \tau=\delta t k_{B} T /\left(a^{2} \zeta\right)$, set to $5 \times 10^{-4}(\delta t=0.045 \mathrm{ps})$ for sufficient accuracy $[23,26,46]$.

The initial DNA state was first constrained in a plane to relax its geometrical parameters, such as stretching, bending along a single strand, and twisting, keeping the writhe of the system null. The geometrical constraint was then released, so that the system relaxed its linking number between helical twist $T w$ and writhe $W r$, as described in Eq. (A6) and reported in Table III. Following the work of Mielke et al.
[55], the latter dynamical quantity can be derived from the discretization of the White's integral expression [57,58],

$$
4 \pi W r=\sum_{j} \sum_{i \neq j}\left(\left(\mathbf{r}_{j+1}-\mathbf{r}_{j}\right) \times\left(\mathbf{r}_{i+1}-\mathbf{r}_{i}\right)\right) \cdot \frac{\left(\mathbf{r}_{j}-\mathbf{r}_{i}\right)}{\left|\mathbf{r}_{j}-\mathbf{r}_{i}\right|^{3}} .
$$

The dot product in Eq. (B2) determines the magnitude of relative nonplanar bending of the segments of the helix axis defined by the pair of axis vectors, $\left(\mathbf{r}_{i+1}-\mathbf{r}_{i}\right)$ and $\left(\mathbf{r}_{j+1}-\mathbf{r}_{j}\right)$, with $\mathbf{r}_{i} \equiv\left(\mathbf{r}_{i}^{(1)}+\mathbf{r}_{i}^{(2)}\right) / 2$. The instantaneous writhe of each substructure is found by summing over all pairs.

\section{APPENDIX C: BIASED MD SIMULATION}

\section{Thermodynamic properties}

The well-tempered variant of the metadynamics (WTmetaD) enhanced sampling technique [30,59] was implemented with the coarse-grained (CG) Brownian simulations of the circular and linear dsDNA, and performed using the version 2.3 of the plugin for free energy calculation, named PLUMED [60]. As shown in Fig. 1(b), we considered the width $\rho_{\max }$ of the denaturation bubble, i.e., the maximal distance between paired bases, as collective variable (CV) to bias the dynamics. We also choose to follow the evolution of the minimal twist angle inside the bubble, $\phi_{\min }=\min _{i \in \text { bubble }} \phi_{i}$, where $\phi_{i}$ is defined as the angle between two consecutive base-pair vectors $\rho_{i}$ and $\rho_{i+1}$. According to the algorithm introduced by Barducci et al. [30], a Gaussian bias potential is deposited every $\tau_{G}$ with height $\omega=\omega_{0} e^{-V(s, t) /(f-1) T}$, where $s$ is the $\mathrm{CV}, \omega_{0}$ is the initial height, $T$ is the temperature of the simulation, $V(s, t)$ the metadynamics time-dependent bias,

$$
V(s, t)=\omega \sum_{t^{\prime}<t} \exp \left[-\frac{\left(s(t)-s\left(t^{\prime}\right)\right)^{2}}{2 \delta^{2}}\right],
$$

and $f \equiv(T+\Delta T) / T$ is the bias factor with $\Delta T$ a parameter with the dimension of a temperature. The resolution of the recovered free energy landscape is determined by the width of the Gaussian $\delta$. We put a restraint-wall potential at large values of $\rho_{\max }$ to prevent the system to escape from the metastable state. We checked that a slight change in the position of the wall did not change significantly the results, particularly the positions of the local minimum and the saddle point, as well as the barrier height measured along the minimal free energy path. To further control the error of the reconstructed landscape, we performed three runs of WT-metaD for each DNA system. The other observables are reconstructed afterwards using the reweighting technique of Bonomi et al. [61]. The different sets of values considered in the WT-metaD simulations are given in Table V.

\section{Dynamical properties}

Building on the accelerated dynamics framework of Hamelberg et al. [32] and more recently Tiwary et al. [24,62], we extended the Metadynamics scope to estimate the mean transition times between the metastable (bubble) and the equilibrium (closed) states observed in the circular and linear 
TABLE V. Linear ( $\ell$ DNA) and circular (cDNA) dsDNA parameters considered in the accelerated dynamics framework. $\delta$ and $\omega_{0}$ refer to the width and the initial height of the Gaussian potentials, respectively. $f^{\text {(therm) }}$, wall ${ }^{\text {(therm) }}, \tau_{G}^{\text {(therm) }}$, and $f^{\text {(dyn) }}, \tau_{G}^{\text {(dyn) }}$, correspond to the bias factor, the location of the restraint-wall potential applied on $\rho_{\max }$ and the bias deposition time in the metadynamics simulations dedicated to the reconstruction of the free energy landscape and the determination of the transition rates, respectively. The symbol (-) means no wall was considered. No restraint-wall potential was applied in the simulations dedicated to the determination of the transition rates.

\begin{tabular}{lccccccc}
\hline \hline & $\delta(\mathrm{nm})$ & $\omega_{0}(\mathrm{~kJ} / \mathrm{mol})$ & $\tau_{G}^{\text {(therm) }}(\mathrm{ps})$ & $\tau_{G}^{\text {(dyn) }}(\mathrm{ps})$ & $f^{\text {(therm) }}$ & $f^{\text {(dyn) }}$ & wall $^{\text {(therm) }}(\mathrm{nm})$ \\
\hline$\ell$ DNA & 0.034 & 3 & 25 & 700 & 6 & 3 & 4.0 \\
DNA $_{0}$ & 0.034 & 3 & 25 & 700 & 6 & 3 & - \\
CDNA $_{1 a / 1 b}$ & 0.034 & 3 & 25 & 700 & 6 & 3 & 3 \\
CDNA $_{2 a / 2 b}$ & 0.034 & 3 & 25 & 700 & 20 & 3 \\
CDNA $_{3 a / 3 b}$ & 0.034 & 3 & 25 & 25 & 4.0 \\
\hline \hline
\end{tabular}

dsDNA. WT-metaD was performed using the width $\rho_{\max }$ of the denaturation bubble as $\mathrm{CV}$. We denote by $\tau$, the mean transition time over the barrier from the metastable state to the closed state, and by $\tau_{M}$, the mean transition time for the metadynamics run. The latter changes as the simulation progresses and is linked to the former through the acceleration factor $\alpha(t) \equiv\left\langle e^{\beta V(s, t)}\right\rangle_{M}=\tau / \tau_{M}(t)$, where the angular brackets $\langle\cdots\rangle_{M}$ denote an average over a metadynamics run confined to the metastable basin, and $V(s, t)$ is the metadynamics timedependent bias. To satisfy the main validity criterions, i.e., 1) to consider a set of CVs able to distinguish between the different metastable states [62], and 2) to avoid depositing bias in the transition state region [24], we check that the statistics of transition times follows a Poisson distribution, and increase the time lag between two successive Gaussian depositions $\tau_{G}=\tau_{G}^{(\text {dyn) }}$, as indicated in Table V. We performed several WT-metaD simulations and stop the simulations when the crossing of the barrier and the Gaussian deposition occur unlikely at the same time. To assess the reliability of the biased simulations, we checked that no bias potential was added to the transition state region during the WT-metaD simulations [62]. We also performed statistical analysis of the distribution of transition times. We performed a two-sample KolmogorovSmirnov (KS) test, which does not require a priori knowledge of the underlying distribution [62]. We tested the null hypothesis that the sample of transition times extracted from the metaD simulations and a large sample of times randomly generated according to the theoretical exponential distribution reflect the same underlying distribution. The null hypothesis is conventionally rejected if the $p$-value $<0.05$. The KS test has been performed as implemented in the software CRAN-R [63].

To take into account the presence of slow non-reactive modes competing with enthalpic contribution, as characterized by the configurational entropic contribution along $\phi_{\min }$, we extended the metadynamics scope discussed above to assess the characteristic times associated with the opening and closure of the long-lived thermally assisted denaturation bubbles when their direct numerical estimation was not feasible. To do so, we considered the convergence of the minimal free energy path (MFEP) and the convergence of the free energy of formation, $\Delta F_{0}^{*}$, defined in terms of the joint probability distribution of the CVs [25,31]:

$$
\Delta F_{0}^{*}=-k_{B} T \log \left(\frac{P_{\mathrm{op}}}{P_{\mathrm{cl}}}\right) .
$$

In Eq. (C2), $P_{\mathrm{cl}}$ and $P_{\mathrm{op}}$ are the probabilities of the closed and open DNA states, respectively. The probability of each state is computed as the integral of the distribution within the energy basin, $\mathcal{B}$, it occupies on the $\mathrm{CV}$-space,

$$
P_{i}=\iint_{\left(\rho_{\max }, \phi_{\min }\right) \in \mathcal{B}_{\mathrm{i}}} f\left(\rho_{\max }, \phi_{\min }\right) d \rho_{\max } d \phi_{\min },
$$

where $f$ is the joint probability density distribution function associated with the system free energy. We considered the successive isosurfaces depicted in Fig. 3 in the main text as integration domains. In every systems, we obtained strong convergence $\left(<0.5 k_{B} T\right)$ of $\Delta F_{0}^{*}$ within four isosurfaces $\left(8 k_{B} T\right)$. Based on the multidimensional Kramers-Langer's framework discussed in the main text, we computed the ratio between the rates associated with the transition between the two free energy basins $\mathcal{B}_{\mathrm{cl}}$ and $\mathcal{B}_{\mathrm{op}}$ shown in Fig. 3:

$$
\frac{k_{\text {opening }}}{k_{\text {closure }}}=\left(\frac{\operatorname{det} \mathbf{H}_{\mathrm{cl}}}{\operatorname{det} \mathbf{H}_{\mathrm{op}}}\right)^{1 / 2} e^{-\Delta F_{0} / k_{B} T} .
$$

In Eq. (C4), $\Delta F_{0}$ represents the free energy of formation between the two free energy basins $\mathcal{B}_{\mathrm{cl}}$ and $\mathcal{B}_{\mathrm{op}}$ measured along the converged MFEP depicted in the main text. We also assumed that the effective friction coefficient remains unchanged on both sides of the transition state, as ascertained from the direct numerical estimations of the characteristic times of $\ell \mathrm{DNA}, \mathrm{cDNA}_{0}, \mathrm{cDNA}_{1 a}$, and $\mathrm{cDNA}_{1 b}$. The parameters $\left(\operatorname{det} \mathbf{H}_{\mathrm{op}}\right)^{1 / 2}$ and $\left(\operatorname{det} \mathbf{H}_{\mathrm{cl}}\right)^{1 / 2}$ represent the effective stiffness of the free energy well associated with the open (op) and closed (cl) dsDNA states, respectively. To account for the asymmetric nature of the free energy landscape in the free energy basins, asymmetric fitting of the free energy surface was considered. The respective values are reported in Table IV. When direct numerical estimation of the characteristic times is achievable $\left(\ell \mathrm{DNA}, \mathrm{cDNA}_{0}, \mathrm{cDNA}_{1 a}\right.$, and $\left.\operatorname{cDNA}_{1 b}\right)$, we checked the ratios $\left(k_{\mathrm{op}} / k_{\mathrm{cl}}\right) /\left(\tau_{\mathrm{cl}} / \tau_{\mathrm{op}}\right)$ are of order 1 , as expected, which supports the validity of our approach to compute both equilibrium free energies and transition rates.
[1] A. Kornberg and T. A. Baker, DNA Replication, 2nd ed. (University Science Books, Sausalito, CA, USA, 1992).
[2] R. Phillips, J. Kondev, J. Theriot, and H. Garcia, Physical Biology of the Cell (Garland Science, New York, USA, 2013). 
[3] J. F. Léger, J. Robert, L. Bourdieu, D. Chatenay, and J. F. Marko, Reca binding to a single double-stranded DNA molecule: A possible role of DNA conformational fluctuations, Proc. Natl. Acad. Sci. USA 95, 12295 (1998).

[4] D. Kowalski, D. A. Natale, and M. J. Eddy, Stable DNA unwinding, not "breathing," accounts for single-strand-specific nuclease hypersensitivity of specific a+t-rich sequences, Proc. Natl. Acad. Sci. USA 85, 9464 (1988).

[5] T. Ambjornsson and R. Metzler, Binding dynamics of singlestranded DNA binding proteins to fluctuating bubbles in breathing DNA, J. Phys.: Condens. Matter 17, S1841 (2005).

[6] P. H. von Hippel, N. P. Johnson, and A. H. Marcus, Fifty years of DNA "breathing": Reflections on old and new approaches, Biopolymers 99, 923 (2013).

[7] N. Destainville, M. Manghi, and J. Palmeri, Microscopic mechanism for experimentally observed anomalous elasticity of DNA in two dimensions, Biophys. J. 96, 4464 (2009).

[8] J. Adamcik, J.-H. Jeon, K. J. Karczewski, R. Metzler, and G. Dietler, Quantifying supercoiling-induced denaturation bubbles in DNA, Soft Matter 8, 8651 (2012).

[9] R. N. Irobalieva, J. M. Fogg, D. J. Catanese Jr., T. Sutthibutpong, M. Chen, A. K. Barker, S. J. Ludtke, S. A. Harris, M. F. Schmid, W. Chiu, and L. Zechiedrich, Structural diversity of supercoiled DNA, Nat. Commun. 6, 8440 (2015).

[10] G. Altan-Bonnet, A. Libchaber, and O. Krichevsky, Bubble Dynamics in Double-Stranded DNA, Phys. Rev. Lett. 90, 138101 (2003).

[11] M. Barbi, S. Cocco, M. Peyrard, and S. Ruffo, A twist model for DNA, J. Biol. Phys. 24, 97 (1999).

[12] M. Barbi, S. Lepri, M. Peyrard, and N. Theodorakopoulos, Thermal denaturation of a helicoidal DNA model, Phys. Rev. E 68, 061909 (2003).

[13] H. C. Fogedby and R. Metzler, DNA Bubble Dynamics as a Quantum Coulomb Problem, Phys. Rev. Lett. 98, 070601 (2007).

[14] R. Metzler, T. Ambjornsson, A. Hanke, and H. C. Fogedby, Single DNA denaturation and bubble dynamics, J. Phys.: Condens. Matter 21, 034111 (2009).

[15] Y. A. G. Fosado, D. Michieletto, and D. Marenduzzo, Dynamical Scaling and Phase Coexistence in Topologically Constrained DNA Melting, Phys. Rev. Lett. 119, 118002 (2017).

[16] S. P. Mielke, N. Grønbech-Jensen, V. V. Krishnan, W. H. Fink, and C. J. Benham, Brownian dynamics simulations of sequence-dependent duplex denaturation in dynamically superhelical DNA, J. Chem. Phys. 123, 124911 (2005).

[17] F. Trovato and V. Tozzini, Supercoiling and local denaturation of plasmids with a minimalist DNA model, J. Phys. Chem. B 112, 13197 (2008).

[18] D. Jost, A. Zubair, and R. Everaers, Bubble statistics and positioning in superhelically stressed DNA, Phys. Rev. E 84, 031912 (2011).

[19] J. P. K. Doye, T. E. Ouldridge, A. A. Louis, F. Romano, P. Sulc, C. Matek, B. E. K. Snodin, L. Rovigatti, J. S. Schreck, R. M. Harrison, and W. P. J. Smith, Coarse-graining DNA for simulations of DNA nanotechnology, Phys. Chem. Chem. Phys. 15, 20395 (2013).

[20] C. Matek, T. E. Ouldridge, J. P. K. Doye, and A. A. Louis, Plectoneme tip bubbles: Coupled denaturation and writhing in supercoiled DNA, Sci. Rep. 5, 7655 (2015).
[21] Y. Shibata, P. Kumar, R. Layer, S. Willcox, J. R. Gagan, J. D. Griffith, and A. Dutta, Extrachromosomal micro DNAs and chromosomal microdeletions in normal tissues, Science 336, 82 (2012).

[22] M. Manghi and N. Destainville, Physics of base-pairing dynamics in DNA, Phys. Rep. 631, 1 (2016).

[23] F. Sicard, N. Destainville, and M. Manghi, DNA denaturation bubbles: Free-energy landscape and nucleation/closure rates, J. Chem. Phys. 142, 034903 (2015).

[24] P. Tiwary and M. Parrinello, From Metadynamics to Dynamics, Phys. Rev. Lett. 111, 230602 (2013).

[25] F. Sicard, Computing transition rates for rare event: When Kramers theory meets the free-energy landscape, Phys. Rev. E 98, 052408 (2018).

[26] A. K. Dasanna, N. Destainville, J. Palmeri, and M. Manghi, Slow closure of denaturation bubbles in DNA: Twist matters, Phys. Rev. E 87, 052703 (2013).

[27] A. Vologodskii, Biophysics of DNA (Cambridge University Press, Cambridge, 2015).

[28] M. Sayar, B. Avsaroglu, and A. Kabakcioglu, Twist-writhe partitioning in a coarse-grained DNA minicircle model, Phys. Rev. E 81, 041916 (2010).

[29] N. P. Higgins and A. V. Vologodskii, Topological behavior of plasmid DNA, Microbiol. Spectr. 3, 105 (2015).

[30] A. Barducci, G. Bussi, and M. Parrinello, Well-Tempered Metadynamics: A Smoothy Converging and Tunable Free-Energy Method, Phys. Rev. Lett. 100, 020603 (2008).

[31] I. Gimondi and M. Salvalaglio, $\mathrm{CO}_{2}$ packing polymorphism under pressure: Mechanism and thermodynamics of the iiii polymorphic transition, J. Chem. Phys. 147, 114502 (2017).

[32] Y. Xin, U. Doshi, and D. Hamelberg, Examining the limits of time reweighting and Kramers' rate theory to obtain correct kinetics from accelerated molecular dynamics, J. Chem. Phys. 132, 224101 (2010).

[33] D. G. Truhlar, B. C. Garrett, and S. J. Klippenstein, Current status of transition-state theory, J. Phys. Chem. 100, 12771 (1996).

[34] B. Peters, Reaction Rate Theory and Rare Events, 1st ed. (Elsevier, Amsterdam, The Netherlands, 2017).

[35] J. S. Langer, Statistical theory of the decay of metastable states, Ann. Phys (NY). 54, 258 (1969).

[36] A. M. Berezhkovskii and V. Yu. Zitserman, Solvent slowmode influence on chemical reaction dynamics: a multidimensional Kramers-theory treatment, Chem. Phys. Lett. 172, 235 (1990).

[37] A. M. Berezhkovskii, V. Yu. Zitserman, and A. Polimeno, Numerical test of Kramers reaction rate theory in two dimensions, J. Chem. Phys. 105, 6342 (1996).

[38] C. Wei and A. Pohorille, Flip-flop of oleic acid in a phospholipid membrane: Rate and mechanism, J. Phys. Chem. B 118, 12919 (2014).

[39] S. W. Englander, N. R. Kallenbach, A. J. Heeger, J. A. Krumhansl, and S. Litwin, Nature of the open state in long polynucleotide double helices: Possibility of soliton excitations, Proc. Nat. Acad. Soc. USA 77, 7222 (1980).

[40] B. Chen, Y. Xiao, C. Liu, C. Li, and F. Leng, DNA linking number change induced by sequence-specific DNA-binding proteins, Nucleic Acids Res. 38, 3643 (2010). 
[41] M. Segal, A. Ingargiola, E. Lerner, S. Chung, J. A. White, A. Streets, S. Weiss, and X. Michalet, High-throughput smFRET analysis of freely diffusing nucleic acid molecules and associated proteins, Methods 169, 21 (2019).

[42] K. Nowak-Lovato, L. B. Alexandrov, A. Banisadr, A. L. Bauer, A. R. Bishop, A. Usheva, F. Mu, E. Hong-Geller, K. Rasmussen, and W. S. Hlavacek, Binding of nucleoidassociated protein fis to DNA is regulated by DNA breathing dynamics, PLoS Comput. Biol. 9, e1002881 (2013).

[43] L. V. Croft, E. Bolderson, M. N. Adams, S. El-Kamand, R. Kariawasam, L. Cubeddu, R. Gamsjaeger, and D. J. Richard, Human single-stranded DNA binding protein 1 (hSSB1, OBFC2B), a critical component of the DNA damage response, Semin. Cell. Dev. Biol. 86, 121 (2019).

[44] Y. Wu, H. Chen, J. Lu, M. Zhang, R. Zhang, T. Duan, X. Wang, J. Huang, and T. Kang, Acetylation-dependent function of human single-stranded DNA binding protein 1, Nucleic Acids Res. 43, 7878 (2015).

[45] T. Hugel, M. Rief, M. Seitz, H. E. Gaub, and R. R. Netz, Highly Stretched Single Polymers: Atomic-Force-Microscope Experiments Versus Ab-Initio Theory, Phys. Rev. Lett. 94, 048301 (2005).

[46] A. K. Dasanna, N. Destainville, J. Palmeri, and M. Manghi, Strand diffusion-limited closure of denaturation bubbles in DNA, Europhys. Lett. 98, 38002 (2012).

[47] B. Tinland, A. Pluen, J. Sturm, and G. Weill, Persistence length of single-stranded DNA, Macromolecules 30, 5763 (1997).

[48] T. E. Cloutier and J. Widom, Spontaneous sharp bending of double-stranded DNA, Mol. Cell 14, 355 (2004).

[49] T. E. Cloutier and J. Widom, DNA twisting flexibility and the formation of sharply looped protein-DNA complexes, Proc. Nat. Acad. Soc. USA 102, 3645 (2005).

[50] L. M. Bond, J. P. Peters, N. A. Becker, J. D. Kahn, and L. J. Maher III, Gene repression by minimal lac loops in vivo, Nucleic Acids Research 38, 8072 (2010).

[51] L. W. Dillon, P. Kumar, Y. Shibata, Y.-H. Wang, S. Willcox, J. D. Griffith, Y. Pommier, S. Takeda, and A. Dutta, Produc- tion of extrachromosomal microdnas is linked to mismatch repair pathways and transcriptional activity, Cell Rep. 11, 1749 (2015).

[52] R. P. Bowater, Supercoiled DNA: Structure (eLS John Wiley \& Sons, Ltd, Chichester, 2015).

[53] K. Olsen and J. Bohr, Geometry of the toroidal $n$-helix: Optimal-packing and zero-twist, New J. Phys. 14, 023063 (2012).

[54] J. F. Marko, Biophysics of protein-DNA interactions and chromosome organization, Physica A 418, 126 (2015).

[55] S. P. Mielke, W. H. Fink, V. V. Krishnan, N. Grønbech-Jensen, and C. J. Benham, Transcription-driven twin supercoiling of a DNA loop: A Brownian dynamics study, J. Chem. Phys. 121, 8104 (2004).

[56] T. Murtola, A. Bunker, I. Vattulainen, M. Deserno, and M. Karttunen, Multiscale modeling of emergent materials: Biological and soft matter, Phys. Chem. Chem. Phys. 11, 1869 (2009).

[57] J. H. White and W. R. Bauer, Calculation of the twist and the writhe for representative models of DNA, J. Mol. Biol. 189, 329 (1986).

[58] J. F. Marko and E. D. Siggia, Statistical mechanics of supercoiled DNA, Phys. Rev. E 52, 2912 (1995).

[59] J. F. Dama, M. Parrinello, and G. A. Voth, Well-Tempered Metadynamics Converges Asymptotically, Phys. Rev. Lett. 112, 240602 (2014).

[60] G. A. Tribello, M. Bonomi, D. Branduardi, C. Camilloni, and G. Bussi, Plumed 2: New feathers for an old bird, Comput. Phys. Comm. 185, 604 (2014).

[61] M. Bonomi, A. Barducci, and M. Parrinello, Reconstructing the equilibrium Boltzmann distribution from welltempered metadynamics, J Comput. Chem. 30, 1615 (2009).

[62] M. Salvalaglio, P. Tiwary, and M. Parrinello, Assessing the reliability of the dynamics reconstructed from metadynamics, J. Chem. Theory Comput. 10, 1420 (2014).

[63] The comprehensive R archive network, https://cran.r-project. org/ (accessed January 17, 2018). 